\title{
Very Compact Hardware Implementations of the Blockcipher CLEFIA
}

\author{
Toru Akishita and Harunaga Hiwatari \\ Sony Corporation \\ 5-1-12 Kitashinagawa Shinagawa-ku, Tokyo 141-0001, Japan \\ \{Toru.Akishita, Harunaga.Hiwatari\}@jp.sony.com
}

\begin{abstract}
The 128-bit blockcipher CLEFIA is known to be highly efficient in hardware implementations. This paper proposes very compact hardware implementations of CLEFIA-128. Our implementations are based on novel serialized architectures in the data processing block. Three types of hardware architectures are implemented and synthesized using a $0.13 \mu \mathrm{m}$ standard cell library. In the smallest implementation, the area requirements are only 2,488 GE, which are about half of the previous smallest implementation as far as we know. Furthermore, only additional $116 \mathrm{GE}$ enable to support decryption.
\end{abstract}

Keywords: blockcipher, CLEFIA, compact hardware implementation, ASIC.

\section{Introduction}

CLEFIA 911] is a 128-bit blockcipher supporting key lengths of 128, 192 and 256 bits, which is compatible with AES [2]. CLEFIA achieves enough immunity against known attacks and flexibility for efficient implementation in both hardware and software. It is reported that CLEFIA is highly efficient particularly in hardware implementations [121013.

Compact hardware implementations are very significant for small embedded devices such as RFID tags and wireless sensor nodes because of their limited hardware resources. As for AES with 128-bit keys, low-area hardware implementations have been reported in 3 and [4. The former uses a RAM based architecture supporting both encryption and decryption with the area requirements of 3,400 GE, while the latter uses a shift-register based architecture supporting encryption only with the area requirements of 3,100 GE. Both implementations use an 8-bit serialized data path and implement only a fraction of the MixColumns operation with additional three 8-bit registers, where it takes several clock cycles to calculate one column. Very recently, another low-area hardware implementation of AES was proposed in [5] requiring 2,400 GE for encryption only. Unlike the previous two implementations, it implements MixColumns not in a serialized way, where one column of MixColumns is processed in 1 clock cycle. Thus it requires 4 times more XOR gates for MixColumns, but requires no additional register and can reduce gate requirements for control logic. 
In this paper, we present very compact hardware architectures of CLEFIA with 128-bit keys based on 8-bit shift registers. We show that the data processing part of CLEFIA-128 can be implemented in a serialized way without any additional registers. Three types of hardware architectures are proposed according to required cycles for one block process by adaptively applying clock gating technique. Those architectures are implemented and synthesized using a 0.13 $\mu \mathrm{m}$ standard cell library. In our smallest implementation, the area requirements are only 2,488 GE, which are to the best of our knowledge about half as small as the previous smallest implementation, 4,950 GE [10 12], and competitive to the smallest AES implementation. Furthermore, only additional 116 GE are required to support decryption by switching the processing order of F-functions at even-numbered rounds.

The rest of the paper is organized as follows. Sect. 2 gives brief description of CLEFIA and its previously proposed hardware implementations. In Sect. 3 , we propose three types of hardware architectures. Sect. 4 describes additional hardware resources to support decryption. Sect. 5] gives evaluation results for our implementations, compared with the previous results of CLEFIA and AES. Finally, we conclude in Sect. 6.

\section{128-bit Blockcipher CLEFIA}

\subsection{Algorithm}

CLEFIA 911 is a 128-bit blockcipher with its key length being 128, 192, and 256 bits. For brevity, we consider 128-bit key CLEFIA, denoted as CLEFIA-128, though similar techniques are applicable to CLEFIA with 192-bit and 256-bit keys. CLEFIA-128 is divided into two parts: the data processing part and the key scheduling part.

The data processing part employs a 4-branch Type-2 generalized Feistel network 14 with two parallel F-functions $F_{0}$ and $F_{1}$ per round. The number of rounds $r$ for CLEFIA-128 is 18 . The encryption function $E N C_{r}$ takes a 128-bit plaintext $P=P_{0}\left|P_{1}\right| P_{2} \mid P_{3}, 32$-bit whitening keys $W K_{i}(0 \leq i<4)$, and 32bit round keys $R K_{j}(0 \leq j<2 r)$ as inputs, and outputs a 128-bit ciphertext $C=C_{0}\left|C_{1}\right| C_{2} \mid C_{3}$ as shown in Fig. 1.

The two F-functions $F_{0}$ and $F_{1}$ consist of round key addition, 4 non-linear 8-bit S-boxes, and a diffusion matrix. The construction of $F_{0}$ and $F_{1}$ is shown in Fig. 2. Two kind of S-boxes $S_{0}$ and $S_{1}$ are employed, and the order of these S-boxes is different in $F_{0}$ and $F_{1}$. The diffusion matrices of $F_{0}$ and $F_{1}$ are also different; the matrices $M_{0}$ for $F_{0}$ and $M_{1}$ for $F_{1}$ are defined as

$$
M_{0}=\left(\begin{array}{llll}
01 & 02 & 04 & 06 \\
02 & 01 & 06 & 04 \\
04 & 06 & 01 & 02 \\
06 & 04 & 02 & 01
\end{array}\right), \quad M_{1}=\left(\begin{array}{llll}
01 & 08 & 02 & 0 \mathrm{~A} \\
08 & 01 & 0 \mathrm{~A} & 02 \\
02 & 0 \mathrm{~A} & 01 & 08 \\
0 \mathrm{~A} & 02 & 08 & 01
\end{array}\right)
$$

The multiplications between these matrices and vectors are performed in $\operatorname{GF}\left(2^{8}\right)$ defined by a primitive polynomial $z^{8}+z^{4}+z^{3}+z^{2}+1$. 


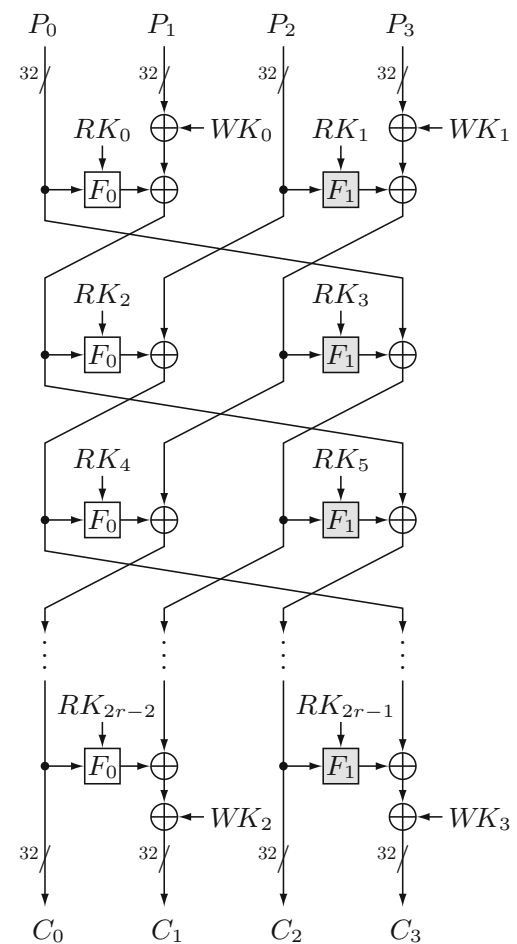

Fig. 1. Encryption function $E N C_{r}$
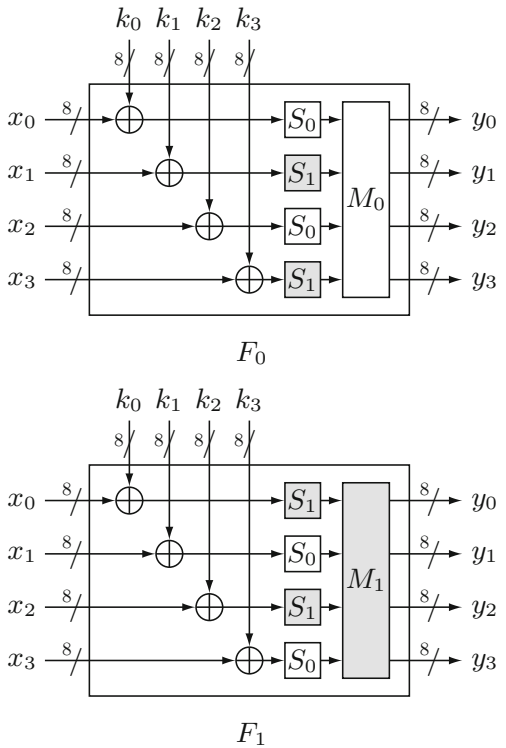

Fig. 2. F-functions $F_{0}, F_{1}$

128 bits

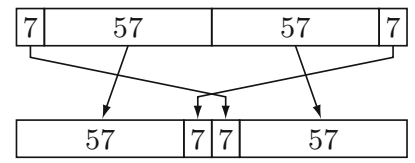

Fig. 3. DoubleSwap function $\Sigma$

The key scheduling part of CLEFIA-128 takes a secret key $K$ as an input, and outputs 32-bit whitening keys $W K_{i}(0 \leq i<4)$ and 32-bit round keys $R K_{j}(0 \leq j<2 r)$. It is divided into the following two steps: generating a 128bit intermediate key $L$ (step 1) and generating $W K_{i}$ and $R K_{j}$ from $K$ and $L$ (step 2 ). In step 1 , the intermediate key $L$ is generated by 12 rounds of encryption function which takes $K$ as a plaintext and constant values $C O N_{i}(0 \leq i<24)$ as round keys. In step 2 , the intermediate key $L$ is updated by the DoubleSwap function $\Sigma$, which is illustrated in Fig. 3. Round keys $R K_{j}(0 \leq j<36)$ is generated by mixing $K, L$, and constant values $C O N_{i}(24 \leq i<60)$. Whitening keys $W K_{i}$ are equivalent to 32 -bit chunks $K_{i}$ of $K$ as $K=K_{0}\left|K_{1}\right| K_{2} \mid K_{3}$.

\subsection{Previous Hardware Implementations}

Hardware implementations of CLEFIA-128 have been studied in [121013. In [12, optimization techniques in data processing part including S-boxes and 
diffusion matrices were proposed. The compact architecture, where $F_{0}$ is processed in one cycle and $F_{1}$ is processed in another cycle, was implemented, and its area requirements in area optimization are reported to be 4,950 GE.

In [10, two optimization techniques in key scheduling part were introduced. The first technique is related to implementation of the DoubleSwap function $\Sigma$. $\Sigma$ is decomposed into the following Swap function $\Omega$ and $S u b S w a p$ function $\Psi$ as $\Sigma=\Psi \circ \Omega$.

$$
\begin{aligned}
\Omega: X & \mapsto Y \\
Y & =X[64-127] \mid X[0-63] \\
\Psi: X & \mapsto Y \\
Y & =X[71-127]|X[57-70]| X[0-56]
\end{aligned}
$$

$X[a-b]$ denotes a bit string cut from the $a$-th bit to the $b$-th bit of $X$. Please note that $\Omega$ and $\Psi$ are both involutive. The 128-bit key register for the intermediate key $L$ is updated by applying $\Omega$ and $\Psi$ alternately. Round keys are always generated from the most significant 64-bit of the key register. After the final round of encryption, $L$ is re-stored into the key register by applying the following FinalSwap function $\Phi$.

$$
\begin{aligned}
\Phi: X \mapsto & Y \\
Y= & X[49-55]|X[42-48]| X[35-41]|X[28-34]| X[21-27]|X[14-20]| \\
& X[7-13]|X[0-6]| X[64-71]|X[56-63]| X[121-127]|X[114-120]| \\
& X[107-113]|X[100-106]| X[93-99]|X[86-92]| X[79-85] \mid X[72-78]
\end{aligned}
$$

Please note that $\Phi$ is also involutive. In case of decryption, round keys are always generated from the most significant 64-bit of the key register by applying the inverse functions of $\Omega, \Psi$ and $\Phi$ in reverse order of encryption. Due to their involutive property, only three functions $\Omega, \Psi$ and $\Phi$ are required for encryption and decryption.

In the second technique, XOR operations with the parts of round keys related to a secret key $K$ are moved by an equivalent transformation into the two data lines where key whitening operations are processed. Therefore, these XOR operations and key whitening operations can be shared.

In 13, five types of hardware architectures were designed and fairly compared to the ISO 18033-3 standard blockciphers under the same conditions. In their results, the highest efficiency of $400.96 \mathrm{Kbps} /$ gates was achieved, which is at least 2.2 times higher than that of the ISO 18033-3 standard blockciphers.

\section{Proposed Architectures}

In this section we propose three types of hardware architectures. Firstly, we propose a compact matrix multiplier for CLEFIA-128. Next, in Type-I architecture, we propose a novel serialized architecture of the data processing block of CLEFIA-128. By adaptively applying clock gating logic to Type-I architecture, 


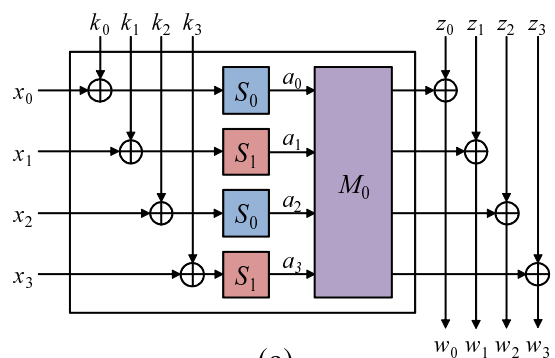

(a)

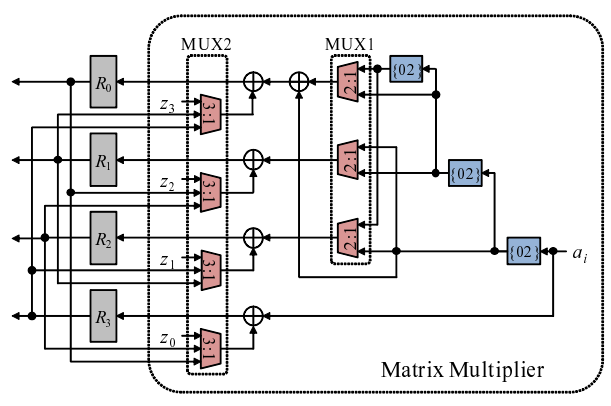

(b)

\begin{tabular}{|c||c|c|c|c|}
\hline$l$ & 1 & 2 & 3 & 4 \\
\hline$R_{0}$ & $z_{3} \oplus\{06\} a_{0}$ & $z_{2} \oplus\{04\} a_{0} \oplus\{06\} a_{1}$ & $z_{1} \oplus\{02\} a_{0} \oplus a_{1} \oplus\{06\} a_{2}$ & $z_{0} \oplus a_{0} \oplus\{02\} a_{1} \oplus\{04\} a_{2} \oplus\{06\} a_{3}$ \\
\hline$R_{1}$ & $z_{2} \oplus\{04\} a_{0}$ & $z_{3} \oplus\{06\} a_{0} \oplus\{04\} a_{1}$ & $z_{0} \oplus a_{0} \oplus\{02\} a_{1} \oplus\{04\} a_{2}$ & $z_{1} \oplus\{02\} a_{0} \oplus a_{1} \oplus\{06\} a_{2} \oplus\{04\} a_{3}$ \\
\hline$R_{2}$ & $z_{1} \oplus\{02\} a_{0}$ & $z_{0} \oplus a_{0} \oplus\{02\} a_{1}$ & $z_{3} \oplus\{06\} a_{0} \oplus\{04\} a_{1} \oplus\{02\} a_{2}$ & $z_{2} \oplus\{04\} a_{0} \oplus\{06\} a_{1} \oplus a_{2} \oplus\{02\} a_{3}$ \\
\hline$R_{3}$ & $z_{0} \oplus a_{0}$ & $z_{1} \oplus\{02\} a_{0} \oplus a_{1}$ & $z_{2} \oplus\{04\} a_{0} \oplus\{06\} a_{1} \oplus a_{2}$ & $z_{3} \oplus\{06\} a_{0} \oplus\{04\} a_{1} \oplus\{02\} a_{2} \oplus a_{3}$ \\
\hline
\end{tabular}

(c)

Fig. 4. Matrix multiplier: (a) $F$-function $F_{0}$, (b) Data path, (c) Contents of registers $R_{j}(0 \leq j<4)$ at the $l$-th cycle

we can reduce the number of multiplexers (MUXes) in Type-II and Type-III architectures with increasing cycle counts.

Clock gating is a power-saving technique used in synchronous circuits. For hardware implementations of blockciphers, it was firstly introduced in [8] as a technique to reduce gate counts and power consumption, and have been applied to KATAN family [1] and AES [5. Clock gating works by taking the enable conditions attached to registers. It can remove feedback MUXes to hold their present state and replace them with clock gating logic. In case that several bits of registers take the same enable conditions, their gate counts will be saved by applying clock gating.

\subsection{Matrix Multiplier}

Among low-area AES implementations, MixColumns matrix operations are computed row by row in [3], while they are computed column by column in [4]. In our architecture, matrix operations are computed column by column in the following way.

The 4-byte output of $M_{0}$ operation is XORed with the next 4-byte data as shown in Fig. 4 (a). The matrix multiplier in Fig. 4 (b) performs the matrix multiplication together with the above XOR operation in 4 clock cycles. Fig. 4 (c) presents the contents of the registers $R_{i}$ at the $l$-th cycle $(1 \leq l \leq 4)$. At the 1 st cycle, the output $a_{0}$ of $S_{0}$ are fed to the multiplier and multiplied by $\{01\},\{02\}$, $\{04\}$, and $\{06\}$. The products are XORed with the data $z_{i}(0 \leq i<4)$, and then the intermediate results are stored in the four registers $R_{j}(0 \leq j<4)$. As each column in $M_{0}$ consists of the same coefficients, the matrix multiplication can be 


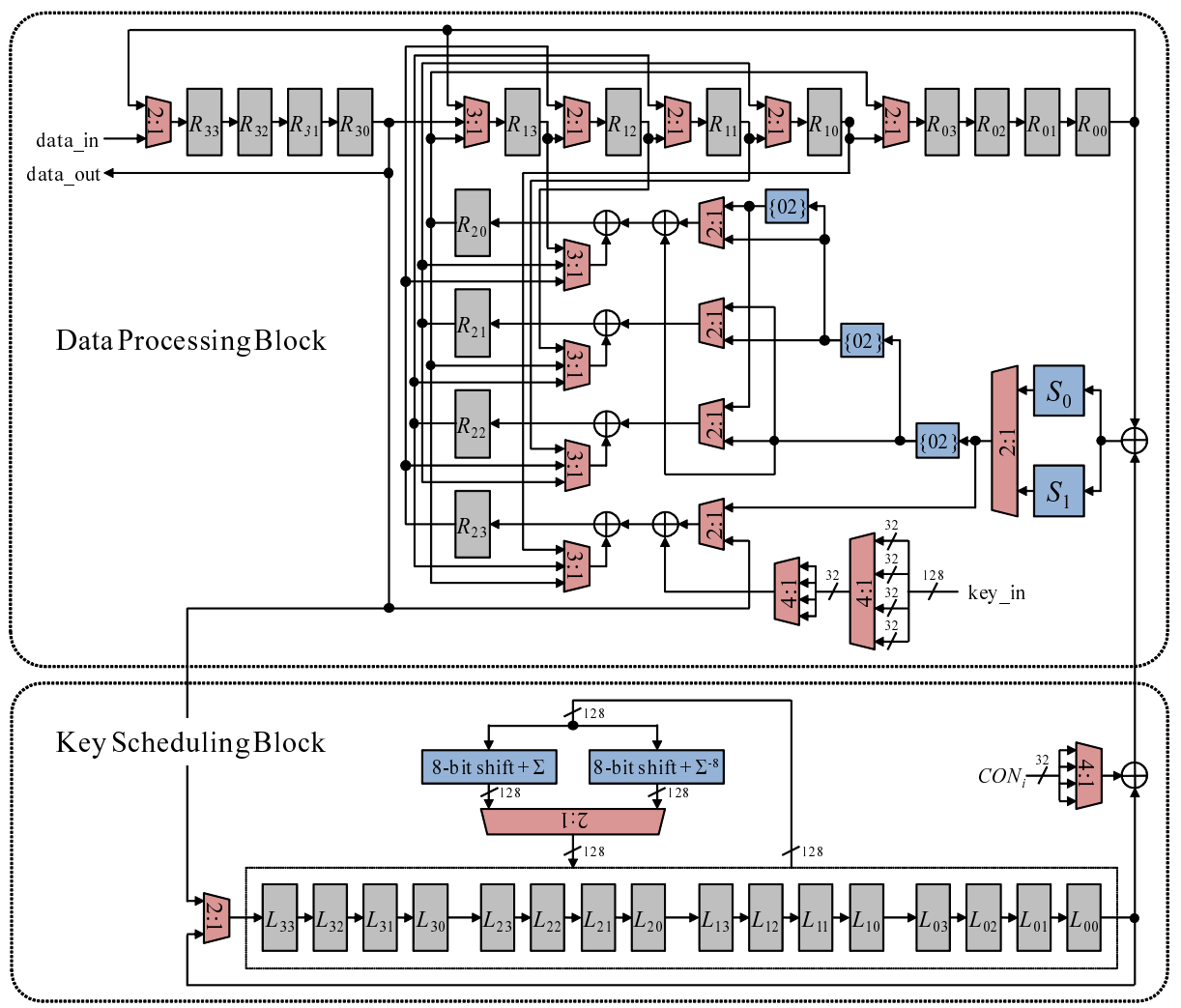

Fig. 5. Data path of Type-I architecture

performed by selecting the intermediate results through MUX2 and XORing the products of $a_{i}(i=1,2,3)$ with them at the $(i+1)$-th cycle. After 4 clock cycles, $w_{i}(0 \leq i<4)$ are stored in $R_{i}$. The multiplication by $M_{1}$ can be performed by switching MUX1.

In 4], three 8-bit registers are required for the construction of a parallel-toserial converter due to avoiding register competition with the next calculation of a matrix. On the other hand, no competition occurs in our architecture because $z_{i}$ is input at the 1 st cycle of a matrix multiplication. $w_{i}$ can be moved into the register where $z_{i}$ for the newly processing F-function is stored.

\subsection{Type-I Architecture}

Fig. 5 shows the data path of Type-I architecture, where the width of data path is 8 bit except those written in the figure. It is divided into the following two blocks: the data processing block and the key scheduling block. Type-I architecture processes a round of the encryption function in 8 clock cycles. We show, in appendix, the detailed data flow of the data registers $R_{i j}(0 \leq i, j<4)$ in Fig. 5 
for a round of the encryption processing. As described in Sect. 3.1, at the 1st and the 5 th cycle in the 8 cycles, the data stored in $R_{20}-R_{23}$ are moved into $R_{03}-R_{12}$, and simultaneously the data stored in $R_{10}-R_{13}$ are input to the matrix multiplier. Therefore, no additional register but the 128-bit data register exists in the data processing block. Please note that $R_{30}-R_{33}$ hold the current state at the $5-8$ th cycle by clock gating.

In the start of encryption, a 128-bit plaintext is located to $R_{i j}$ in 16 clock cycles by inputting it byte by byte from data_in. After 18 rounds of the encryption function which require 144 cycles, a 128-bit ciphertext is output byte by byte from data_out in 16 clock cycles. Therefore, it takes 176 cycles for encryption. The reason why data_out is connected to $R_{30}$ is that no word rotation is necessary at the final round of encryption. In the start of key setup, a 128-bit secret key $K$ input from key_in is located to $R_{i j}$ in 16 clock. After 12 rounds of the encryption function which require 96 cycles, a 128-bit intermediate key $L$ is stored into the key registers $L_{i j}(0 \leq i, j<4)$ by shifting $R_{i j}$ and $L_{i j}$ in 16 clock cycles. Therefore, it takes 128 cycles for key setup.

The two S-box circuits $S_{0}$ and $S_{1}$ are located in the data processing block, and one of those outputs is selected by a 2-to-1 MUX (8-bit width) and input to the matrix multiplier. The encryption processing of CLEFIA-128 is modified by a equivalent transformation as shown in Fig. 7 (a). The 32-bit XOR operation with 32 -bit chunks $K_{i}$ is reduced to the 8 -bit XOR operation by locating it in the matrix multiplier. The 32 -bit chunk $K_{i}$ selected by a 32 -bit 4 -to- 1 MUX is divided into four 8-bit data, and then one of the data is selected by a 8-bit 4-to-1 MUX and fed into the matrix multiplier one by one in 4 clock cycles.

In the key scheduling block, the intermediate key $L$ stored in $L_{i j}$ is cyclically shifted by one byte, and the 8-bit chunk in $L_{00}$ is fed into the data processing after being XORed with the 8-bit chunk of $C O N_{i}$. At the end of even-numbered rounds, $L_{i j}$ is updated by (8-bit shift $+\Sigma$ ) operation; at the end of encryption, $L_{i j}$ is updated by (8-bit shift $+\Sigma^{-8}$ ) operation in order to recover the intermediate key $L$. After restoring the intermediate key $L, L_{i j}$ holds it by clock gating until next start of encryption.

\subsection{Type-II Architecture}

In Type-II architecture, we aim the area optimization of the key scheduling block. Since DoubleSwap function $\Sigma$ is decomposed as $\Sigma=\Psi \circ \Omega$, where $\Psi$ and $\Omega$ are both involutive, as described in Sect. 2.2, $\Sigma^{-8}$ satisfies the following equations.

$$
\begin{aligned}
\Sigma^{-8} & =(\Psi \circ \Omega)^{-8} \\
& =(\Omega \circ \Psi)^{8} \\
& =(\Omega \circ \Psi)^{8} \circ(\Omega \circ \Omega) \\
& =(\Omega \circ \Psi) \circ \cdots \circ(\Omega \circ \Psi) \circ(\Omega \circ \Omega) \\
& =\Omega \circ(\Psi \circ \Omega)^{8} \circ \Omega \\
& =\Omega \circ \Sigma^{8} \circ \Omega
\end{aligned}
$$




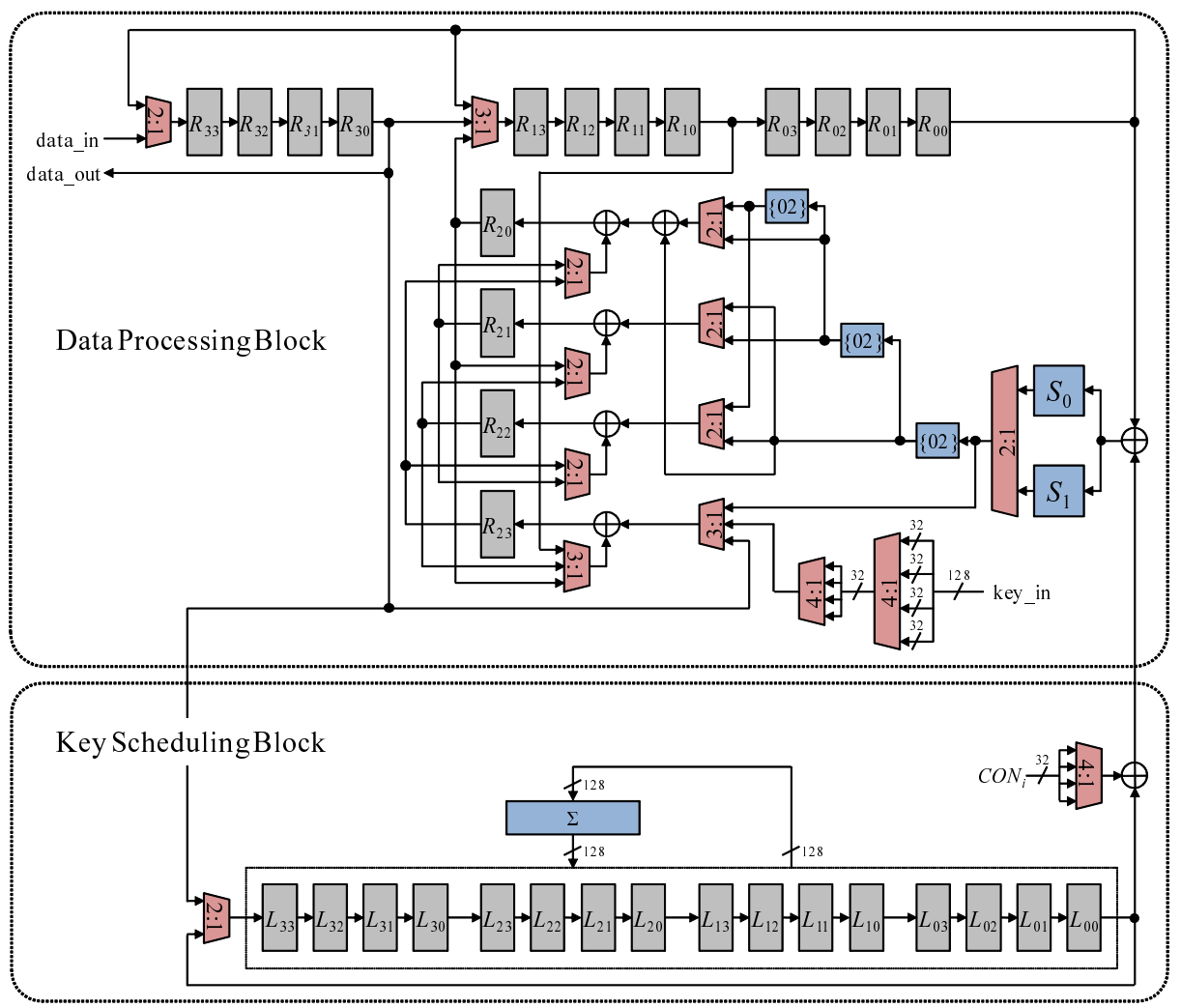

Fig. 6. Data path of Type-III architecture

Swap function $\Omega$ is realized by 8 iterations of cyclic shifting. Thus $\Sigma^{-8}$ operation can be achieved by 8 iterations of cyclic shifting, 8 iterations of $\Sigma$ operation, and 8 iterations of cyclic shifting again, which require 24 cycle counts.

During the encryption processing the intermediate key $L$ is updated by $\Sigma$ operation at the 17 th cycle after 16 iterations of cyclic shifting every two rounds. At the 17th cycle, the data registers must hold the current data by clock gating. Accordingly, both 8 additional cycles for the encryption processing and 8 additional cycles to recover the intermediate key $L$ after outputting a ciphertext are required, which results in 192 cycles for encryption. In compensation for the increase of 16 cycle counts, a 128-bit input of MUX in the key scheduling block can be discarded.

\subsection{Type-III Architecture}

In Type-III architecture, we achieve the area optimization of the data processing block by applying clock gating effectively. Fig. 6 shows the data path of Type-III architecture. Instead of using MUXes, the data stored in $R_{10}-R_{13}$ and 


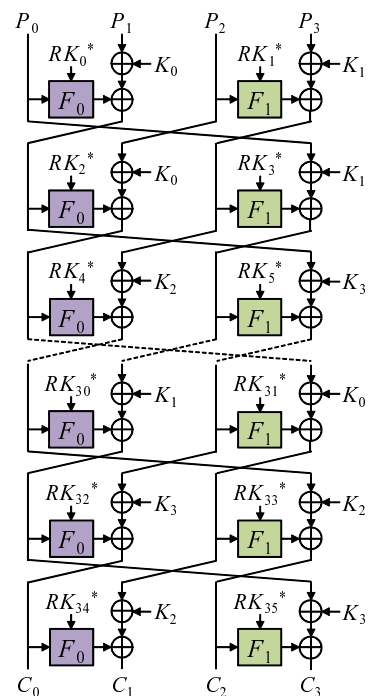

(a)

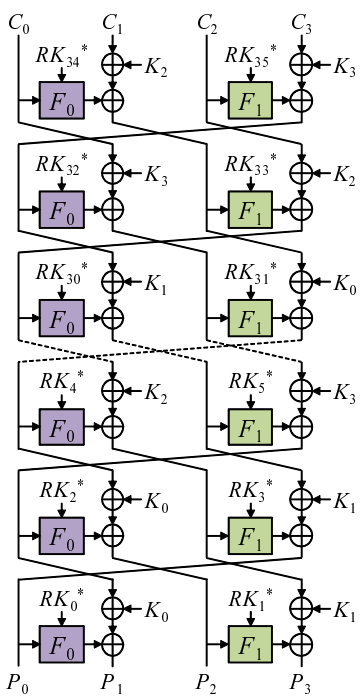

(b)
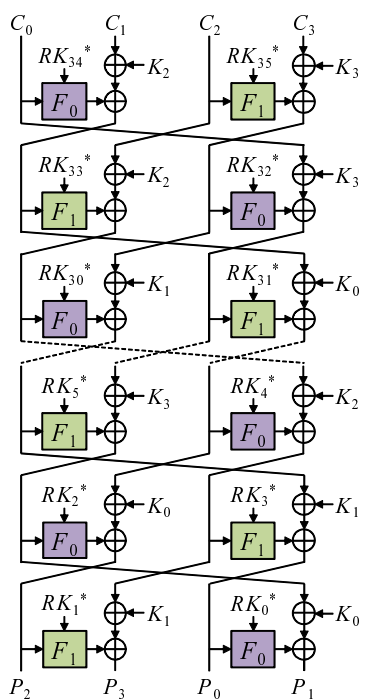

(c)

Fig. 7. (a) Encryption processing, (b) Decryption processing, (c) Optimized decryption processing. XOR operations with the part of round keys related to secret key $K$ are moved by an equivalent transformation, and thus $R K_{j}^{*}(0 \leq j<36)$ denote the remaining part of round keys.

those stored in $R_{20}-R_{23}$ are swapped by cyclically shifting these registers in 4 clock cycles, while the other data register and the key registers hold the current state by clock gating. Simultaneously, the XOR operation with a 32-bit chunk $K_{i}$ is done by XOR gates in the matrix multiplier, which leads the savings of 8 XOR gates. These data swaps are required twice for a round of the encryption processing. Therefore, it takes 16 cycles for a round of the encryption processing; in total 328 and 224 clock cycles are required for encryption and key setup, respectively. In compensation for the increase of many cycle counts, several 8-bit inputs of MUXes together with 8 XOR gates for secret key chunk can be discarded.

\section{Supporting Decryption}

Any encryption-only implementation can support decryption by using the CTR mode. Yet, if the implementation itself supports decryption, it can be used for more application, e.g., an application requiring the $\mathrm{CBC}$ mode. Accordingly, we consider the three types of hardware architectures supporting decryption.

Since the data processing part of CLEFIA employs a 4-branch Type-2 generalized Feistel network [14, the directions of word rotation are different between the encryption function and the decryption function. The encryption and 

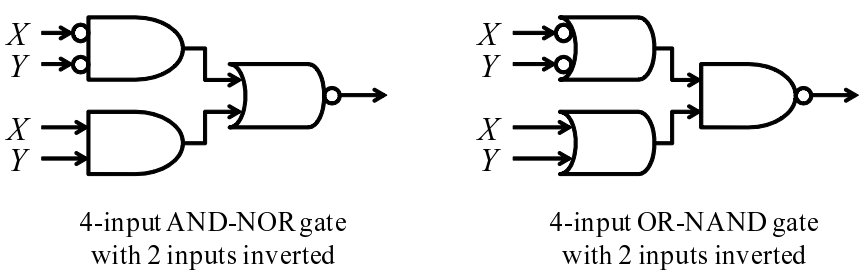

Fig. 8. 4-input AND-NOR and 4-input OR-NAND gate with 2 inputs inverted, which correspond to XOR and XNOR gate

decryption processing of CLEFIA-128 is shown in Fig. 77(a) and (b), respectively. When the hardware architectures described in Sect. 3 support the decryption processing straightforwardly, many additional multiplexers are considered to be required due to these different directions of word rotation. For avoiding this, we switch the positions of $F_{0}$ and those of $F_{1}$ at even-numbered rounds as shown in Fig. 7 (c), and then the direction of word rotation becomes the same as the encryption processing shown in Fig. 7(a). Thus we do not have to largely modify the data path of the above three architectures by processing $F_{1}$ ahead of $F_{0}$ at even-numbered rounds. However, as the order of round keys fed into the data processing block has been changed, the 8-bit round keys are fed from $L_{10}$ when $F_{1}$ is processed at even-numbered rounds and from $L_{30}$ when $F_{0}$ is processed at even-numbered rounds. Accordingly, a 8-bit 3-to-1 MUX is required for selecting the source registers of appropriate round keys including $L_{00}$. Since the leading byte of a ciphertext is stored in $R_{10}$, not $R_{30}$ for encryption, at the end of decryption because of the optimized decryption processing, a 8-bit 2-to-1 MUX is required for selecting data_out.

\section{Implementation Results}

We designed and evaluated the three types of hardware architectures presented in Sect. 3 together with their versions supporting both encryption and decryption. The environment of our hardware design and evaluation is as follows:

$\begin{array}{ll}\text { Language } & \text { Verilog-HDL } \\ \text { Design library } & 0.13 \mu \mathrm{m} \text { CMOS ASIC library } \\ \text { Simulator } & \text { VCS version 2006.06 } \\ \text { Logic synthesis Design Compiler version 2007.03-SP3 }\end{array}$

One Gate Equivalent (GE) is equivalent to the area of a 2-way NAND with the lowest drive strength. For synthesis, we use a clock frequency of $100 \mathrm{KHz}$, which is widely used operating frequency for RFID applications.

Recently, scan flip-flops have been used in the low-area implementations of blockciphers instead of combinations of D flip-flops and 2-to-1 MUXes [815] to reduce area requirements. In our evaluation, a D flip-flop and a 2-to-1 MUX cost 
Table 1. Detailed implementation figures

\begin{tabular}{|l|c|l|c|}
\hline Components [GE] & Type-I & Type-II & Type-III \\
\hline \hline Data Processing Block & 1392.5 & 1392.5 & 1314.5 \\
Data Register (including MUX) & 668 & 668 & 612 \\
S-box (including MUX) & 332.5 & 332.5 & 332.5 \\
S0 & 117.25 & 117.25 & 117.25 \\
S1 & 201.25 & 201.25 & 201.25 \\
Matrix Multiplier & 212 & 212 & 200 \\
Secret Key MUX & 136 & 136 & 136 \\
Secret Key XOR & 16 & 16 & $0 *$ \\
Round Key XOR & 16 & 16 & 16 \\
Other MUX & 12 & 12 & 18 \\
\hline Key Scheduling Block & 952 & 824 & 824 \\
Key Register (including MUX) & 936 & 808 & 808 \\
CON XOR & 16 & 16 & 16 \\
\hline Controller & 333 & 377.25 & 349.25 \\
\hline Total [GE] & 2677.5 & 2593.75 & 2487.75 \\
\hline Cycles [clk] & 176 & 192 & 328 \\
\hline Throughput @100KHz [Kbps] & 73 & 67 & 39 \\
\hline * Secret key XOR is merged to XOR gates in matrix multiplier
\end{tabular}

4.5 and $2.0 \mathrm{GE}$, respectively, while a scan flip-flop costs $6.25 \mathrm{GE}$. Thus, we can save $0.25 \mathrm{GE}$ per bit of storage. Moreover, the library we used has the 4-input AND-NOR and 4-input OR-NAND gates with 2 inputs inverted described in Fig. 8. The outputs of these cells are corresponding to those of XOR or XNOR gates when the inputs $X, Y$ are set as shown in Fig. 8. Since these cells cost 2 GE instead of $2.25 \mathrm{GE}$ required for XOR or XNOR cell, we can save $0.25 \mathrm{GE}$ per XOR or XNOR gate. Clock gating logics are inserted into the design manually by instantiating Integrated Clock Gating (ICG) cells to gate the clocks of specific registers.

Table 1 shows the detailed implementations figures of the three types of hardware architectures presented in Sect. 3. CON generator and selector, ICG cells, and buffers are included in controller.

The area savings for the key scheduling block of Type-II/III implementation over Type-I implementation are $128 \mathrm{GE}$. In the library we used, a register with a 3-to-1 MUX costs 7.25 GE per bit; a register with a 4-to-1 MUX costs $8.25 \mathrm{GE}$ per bit. The key register of Type-I implementation consists of 120 registers with a 3-to-1 MUX (870 GE) and 8 registers with a 4-to-1 MUX (66 GE), while the key register of Type-II/III implementation consists of 120 scan flip-flops (750 GE) and 8 registers with a 3 -to- 1 MUX (58 GE). Thus, the area savings of 128 GE are achieved.

The area savings for the data processing block of Type-III implementation over Type-I/II implementation are $78 \mathrm{GE}$. As for the data register of TypeIII implementation 32 scan flips-flops (200 GE) is replaced with 32 D flip-flops (144 GE), which leads savings of 56 GE. 24 3-to-1 MUXes with output inverted (54 GE) can be replaced with 24 2-to-1 MUXes with output inverted (42 GE) 
Table 2. Implementation results and comparison

\begin{tabular}{|c|c|c|c|c|c|c|}
\hline Algorithm & Source & Mode & $\begin{array}{c}\text { Cycles } \\
{[\mathrm{clk}]}\end{array}$ & $\begin{array}{l}\text { Area } \\
{[\mathrm{GE}]}\end{array}$ & $\begin{array}{c}\text { Throughput @ } 100 \mathrm{KHz} \\
{[\mathrm{Kbps}]}\end{array}$ & $\begin{array}{c}\text { Technology } \\
{[\mu \mathrm{m}]} \\
\end{array}$ \\
\hline \multirow{7}{*}{ CLEFIA } & \multirow{2}{*}{ Type-I } & Enc & 17176 & 2,678 & 73 & \multirow{6}{*}{0.13} \\
\hline & & Enc/Dec & 176 & 2,781 & 73 & \\
\hline & \multirow{2}{*}{ Type-II } & \begin{tabular}{|l|} 
Enc \\
\end{tabular} & 192 & 2,594 & 67 & \\
\hline & & Enc/Dec & $192 / 184$ & 2,678 & $67 / 70$ & \\
\hline & \multirow{2}{*}{ Type-III } & \begin{tabular}{|l|} 
Enc \\
\end{tabular} & 328 & 2,488 & 39 & \\
\hline & & Enc/Dec & $328 / 320$ & 2,604 & $39 / 40$ & \\
\hline & \begin{tabular}{ll|l|l|}
10 & 12 \\
\end{tabular} & Enc/Dec & 36 & 4,950 & 356 & 0.09 \\
\hline \multirow{3}{*}{ AES } & [3] & Enc/Dec & $1,032 / 1,165$ & 3,400 & $12 / 11$ & 0.35 \\
\hline & 4 & \begin{tabular}{|l|} 
Enc \\
\end{tabular} & 177 & 3,100 & 72 & 0.13 \\
\hline & 5 & Enc & 226 & 2,400 & 57 & 0.18 \\
\hline
\end{tabular}

in the matrix multiplier, leading to savings of $12 \mathrm{GE}$. In addition, 8 XOR gates (16 GE) for secret key XOR is merged to XOR gates in the matrix multiplier. Therefore, the area savings of $78 \mathrm{GE}$ are achieved despite the additional $6 \mathrm{GE}$ for the other MUX.

Table 2 shows the implementation results of the proposed architectures together with their versions supporting both encryption and decryption. We also show, for comparison, the best known result of CLEFIA and low-area implementation results of AES. Our implementations supporting encryption only achieve 46-50\% reduction of the area requirements compared to the smallest implementation [1012] of CLEFIA. As for implementations supporting both encryption and decryption, our implementations are 44-47\% smaller. Type-III implementation is $4 \%$ larger than the smallest encryption-only implementation [5] of AES, but its encryption/decryption version achieves $23 \%$ reduction of the area requirements compared to the smallest encryption/decryption implementation [3] of AES.

In order to investigate the components of $47 \%$ area reduction of Type-III implementation supporting both encryption and decryption over 1012 , we first optimized and synthesized the smallest design in [1012] using the ASIC library in this paper. Next, we designed and evaluated Type-I architecture without using hardware implementation technique such as clock gating and the use of scan flipflops. As a result, 10\%, 29\%, and $8 \%$ area reduction was shown to be achieved by the difference of ASIC libraries, data-path serialization, and hardware implementation techniques, respectively. $29 \%$ area reduction by data-path serialization in detail was divided into $8 \%$ by the S-box circuit, $5 \%$ by the matrix multiplier circuit, $6 \%$ by the reduction of XORs, and $10 \%$ by the reduction of MUXes. On the other hand, $8 \%$ area reduction by hardware implementation techniques was divided into $6 \%$ by clock gating, $1 \%$ by the use of scan flip-flops, and $1 \%$ by the other techniques. 


\section{Conclusion}

In this paper, we have proposed very compact hardware architectures of CLEFIA with 128-bit keys based on 8-bit shift registers. We showed that the data processing part of CLEFIA-128 can be implemented in a serialized way without any additional registers. Three types of hardware architectures were proposed according to required cycles for one block process by adaptively applying clock gating technique. Those architectures were implemented and synthesized using a $0.13 \mu \mathrm{m}$ standard cell library. In our smallest implementation, the area requirements are only 2,488 GE, which is $50 \%$ smaller than the smallest implementation of CLEFIA-128, and competitive to the smallest AES-128 implementation. Moreover, the area requirements for its version supporting both encryption and decryption are only $2,604 \mathrm{GE}$, which achieve $23 \%$ reduction of area requirement compared to the smallest encryption/decryption implementation of AES-128.

Future work will include the application of side-channel countermeasures such as threshold implementations [6]7] to the proposed architectures.

\section{References}

1. De Cannière, C., Dunkelman, O., Knežević, M.: KATAN and KTANTAN — A Family of Small and Efficient Hardware-Oriented Block Ciphers. In: Clavier, C., Gaj, K. (eds.) CHES 2009. LNCS, vol. 5747, pp. 272-288. Springer, Heidelberg (2009)

2. Daemen, J., Rijmen, V.: The Design of Rijndael: AES - The Advanced Encryption Standard (Information Security and Cryptography). Springer, Heidelberg (2002)

3. Feldhofer, M., Wolkerstorfer, J., Rijmen, V.: AES Implementation on a Grain of Sand. In: IEE Proceedings Information Security, vol. 152, pp. 13-20 (2005)

4. Hämäläinen, P., Alho, T., Hännikäinen, M., Hämäläinen, T.: Design and Implementation of Low-Area and Low-Power AES Encryption Hardware Core. In: DSD 2006, pp. 577-583. IEEE Computer Society (2006)

5. Moradi, A., Poschmann, A., Ling, S., Paar, C., Wang, H.: Pushing the Limits: A Very Compact and a Threshold Implementation of AES. In: Paterson, K.G. (ed.) EUROCRYPT 2011. LNCS, vol. 6632, pp. 69-88. Springer, Heidelberg (2011)

6. Nikova, S., Rechberger, C., Rijmen, V.: Threshold Implementations against SideChannel Attacks and Glitches. In: Ning, P., Qing, S., Li, N. (eds.) ICICS 2006. LNCS, vol. 4307, pp. 529-545. Springer, Heidelberg (2006)

7. Nikova, S., Rijmen, V., Schläffer, M.: Secure Hardware Implementation of Nonlinear Functions in the Presence of Glitches. In: Lee, P.J., Cheon, J.H. (eds.) ICISC 2008. LNCS, vol. 5461, pp. 218-234. Springer, Heidelberg (2009)

8. Rolfes, C., Poschmann, A., Leander, G., Paar, C.: Ultra-Lightweight Implementations for Smart Devices - Security for 1000 Gate Equivalents. In: Grimaud, G., Standaert, F.-X. (eds.) CARDIS 2008. LNCS, vol. 5189, pp. 89-103. Springer, Heidelberg (2008)

9. Shirai, T., Shibutani, K., Akishita, T., Moriai, S., Iwata, T.: The 128-Bit Blockcipher CLEFIA (Extended Abstract). In: Biryukov, A. (ed.) FSE 2007. LNCS, vol. 4593, pp. 181-195. Springer, Heidelberg (2007)

10. Shirai, T., Shibutani, K., Akishita, T., Moriai, S., Iwata, T.: Hardware Implementations of the 128-bit Blockcipher CLEFIA, Technical Report of IEICE, 107(141), ISEC2007-49, 29-36 (2007) (in Japanese) 
11. The 128-bit Blockcipher CLEFIA: Algorithm Specification, Revision 1.0 (2007), Sony Corporation,

http://www.sony.net/Products/cryptography/clefia/download/

12. The 128-bit Blockcipher CLEFIA: Security and Performance Evaluations, Revision 1.0 (2007), Sony Corporation, http://www.sony.net/Products/cryptography/clefia/download/ download/data/clefia-eval-1.0.pdf

13. Sugawara, T., Homma, N., Aoki, T., Satoh, A.: High-Performance ASIC Implementations of the 128-bit Block Cipher CLEFIA. In: ISCAS 2008, pp. 2925-2928 (2008)

14. Zheng, Y., Matsumoto, T., Imai, H.: On the Construction of Block Ciphers Provably Secure and not Relying on Any Unproved Hypotheses. In: Brassard, G. (ed.) CRYPTO 1989. LNCS, vol. 435, pp. 461-480. Springer, Heidelberg (1990)

\section{Appendix}

In this appendix, we show the detailed data flow of the registers $R_{i j}$ in Fig. 5 during a round of the encryption processing for Type-I architecture. Fig. 9 defines the data structure of a round of the encryption processing. The contents of the registers $R_{i j}(0 \leq i<4)$ are clarified in Table 3 .

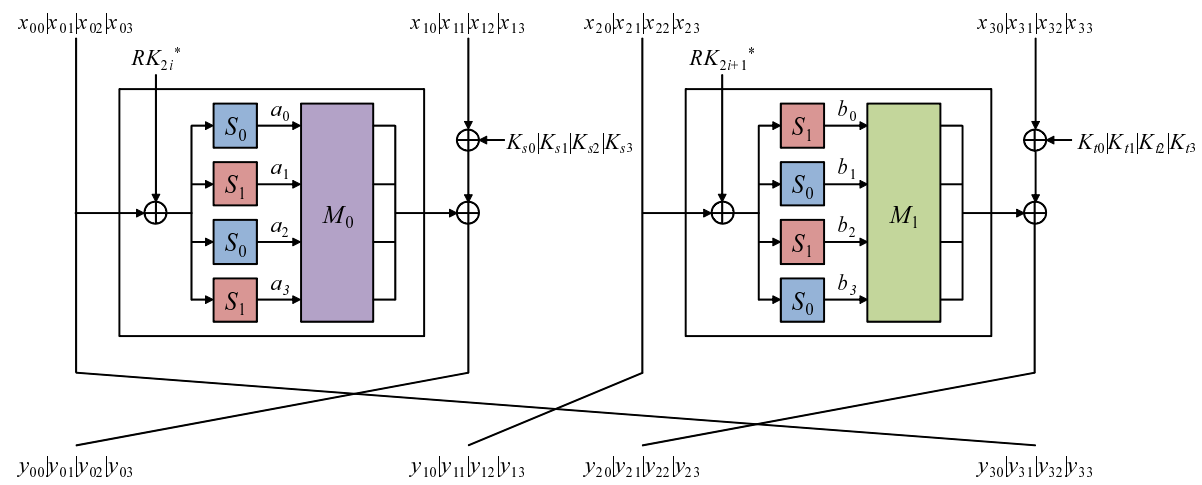

Fig. 9. A round of encryption processing 
Table 3. Contents of registers $R_{i j}(0 \leq i, j<4)$ at the $l$-th cycle

\begin{tabular}{|c|c|c|c|c|c|}
\hline$l$ & 0 & 1 & 2 & 3 & 4 \\
\hline$R_{00}$ & $x_{00}$ & $x_{01}$ & $x_{02}$ & $x_{03}$ & $x_{20}$ \\
\hline$R_{01}$ & $x_{01}$ & $x_{02}$ & $x_{03}$ & $x_{20}$ & $x_{21}$ \\
\hline$R_{02}$ & $x_{02}$ & $x_{03}$ & $x_{20}$ & $x_{21}$ & $x_{22}$ \\
\hline$R_{03}$ & $x_{03}$ & $x_{20}$ & $x_{21}$ & $x_{22}$ & $x_{23}$ \\
\hline$R_{10}$ & $x_{10}$ & $x_{21}$ & $x_{22}$ & $x_{23}$ & $x_{30}$ \\
\hline$R_{11}$ & $x_{11}$ & $x_{22}$ & $x_{23}$ & $x_{30}$ & $x_{31}$ \\
\hline$R_{12}$ & $x_{12}$ & $x_{23}$ & $x_{30}$ & $x_{31}$ & $x_{32}$ \\
\hline$R_{13}$ & $x_{13}$ & $x_{30}$ & $x_{31}$ & $x_{32}$ & $x_{33}$ \\
\hline$R_{20}$ & $x_{20}$ & $x_{13} \oplus\{06\} a_{0}$ & $x_{12} \oplus\{04\} a_{0} \oplus\{06\} a_{1}$ & $x_{11} \oplus\{02\} a_{0} \oplus a_{1} \oplus\{06\} a_{2} \oplus K_{s 1}$ & $y_{00}$ \\
\hline$R_{21}$ & $x_{21}$ & $x_{12} \oplus\{04\} a_{0}$ & $x_{13} \oplus\{06\} a_{0} \oplus\{04\} a_{1}$ & $x_{10} \oplus a_{0} \oplus\{02\} a_{1} \oplus\{04\} a_{2} \oplus K_{s 0}$ & $y_{01}$ \\
\hline$R_{22}$ & $x_{22}$ & $x_{11} \oplus\{02\} a_{0}$ & $x_{10} \oplus a_{0} \oplus\{02\} a_{1} \oplus K_{s 0}$ & $x_{13} \oplus\{06\} a_{0} \oplus\{04\} a_{1} \oplus\{02\} a_{2}$ & $y_{02}$ \\
\hline$R_{23}$ & $x_{23}$ & $x_{10} \oplus a_{0} \oplus K_{s 0}$ & $x_{11} \oplus\{02\} a_{0} \oplus a_{1} \oplus K_{s 1}$ & $x_{12} \oplus\{04\} a_{0} \oplus\{06\} a_{1} \oplus a_{2} \oplus K_{s 2}$ & $y_{03}$ \\
\hline$R_{30}$ & $x_{30}$ & $x_{31}$ & $x_{32}$ & $x_{33}$ & $x_{00}\left(=y_{30}\right)$ \\
\hline$R_{31}$ & $x_{31}$ & $x_{32}$ & $x_{33}$ & $x_{00}$ & $x_{01}\left(=y_{31}\right)$ \\
\hline$R_{32}$ & $x_{32}$ & $x_{33}$ & $x_{00}$ & $x_{01}$ & $x_{02}\left(=y_{32}\right)$ \\
\hline$R_{33}$ & $x_{33}$ & $x_{00}$ & $x_{01}$ & $x_{02}$ & $x_{03}\left(=y_{33}\right)$ \\
\hline $\begin{array}{ll}l \\
\end{array}$ & 4 & $\overline{5}$ & 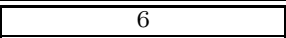 & 7 & 8 \\
\hline$R_{00}$ & $x_{20}$ & $x_{21}$ & $x_{22}$ & $x_{23}$ & $y_{00}$ \\
\hline$R_{01}$ & $x_{21}$ & $x_{22}$ & $x_{23}$ & $y_{00}$ & $y_{01}$ \\
\hline$R_{02}$ & $x_{22}$ & $x_{23}$ & $y_{00}$ & $y_{01}$ & $y_{02}$ \\
\hline$R_{03}$ & $x_{23}$ & $y_{00}$ & $y_{01}$ & $y_{02}$ & $y_{03}$ \\
\hline$R_{10}$ & $x_{30}$ & $y_{01}$ & $y_{02}$ & $y_{03}$ & $x_{20}\left(=y_{10}\right)$ \\
\hline$R_{11}$ & $x_{31}$ & $y_{02}$ & $y_{03}$ & $x_{20}$ & $x_{21}\left(=y_{11}\right)$ \\
\hline$R_{12}$ & $x_{32}$ & $y_{03}$ & $x_{20}$ & $x_{21}$ & $x_{22}\left(=y_{12}\right)$ \\
\hline$R_{13}$ & $x_{33}$ & $x_{20}$ & $x_{21}$ & $x_{22}$ & $x_{23}\left(=y_{13}\right)$ \\
\hline$R_{20}$ & $y_{00}$ & $x_{33} \oplus\{0 \mathrm{~A}\} b_{0}$ & $x_{32} \oplus\{02\} b_{0} \oplus\{0 \mathrm{~A}\} b_{1}$ & $x_{31} \oplus\{08\} b_{0} \oplus b_{1} \oplus\{0 \mathrm{~A}\} b_{2} \oplus K_{t 1}$ & $y_{20}$ \\
\hline$R_{21}$ & $y_{01}$ & $x_{32} \oplus\{02\} b_{0}$ & $x_{33} \oplus\{0 \mathrm{~A}\} b_{0} \oplus\{02\} b_{1}$ & $x_{30} \oplus b_{0} \oplus\{08\} b_{1} \oplus\{02\} b_{2} \oplus K_{t 0}$ & $y_{21}$ \\
\hline$R_{22}$ & $y_{02}$ & $x_{31} \oplus\{08\} b_{0}$ & $x_{30} \oplus b_{0} \oplus\{08\} b_{1} \oplus K_{t 0}$ & $x_{33} \oplus\{0 \mathrm{~A}\} b_{0} \oplus\{02\} b_{1} \oplus\{08\} b_{2}$ & $y_{22}$ \\
\hline$R_{23}$ & $y_{03}$ & $x_{30} \oplus b_{0} \oplus K_{t 0}$ & $x_{31} \oplus\{08\} b_{0} \oplus b_{1} \oplus K_{t 1}$ & $x_{32} \oplus\{02\} b_{0} \oplus\{0 \mathrm{~A}\} b_{1} \oplus b_{2} \oplus K_{t 2}$ & $y_{23}$ \\
\hline$R_{30}$ & $y_{30}$ & $y_{30}$ & $y_{30}$ & $y_{30}$ & $y_{30}$ \\
\hline$R_{31}$ & $y_{31}$ & $y_{31}$ & $y_{31}$ & $y_{31}$ & $y_{31}$ \\
\hline$R_{32}$ & $y_{32}$ & $y_{32}$ & $y_{32}$ & $y_{32}$ & $y_{32}$ \\
\hline$R_{33}$ & $y_{33}$ & $y_{33}$ & $y_{33}$ & $y_{33}$ & $y_{33}$ \\
\hline
\end{tabular}

